\title{
Electron's Orbital Motion Dominates a Spintronic Effect
}

\author{
In a two-dimensional material, the orbital motion of electrons, rather \\ than their spin, is the dominant contribution to an effect harnessed by \\ spintronic devices.
}

By Matteo Rini

$\square$ pintronic devices-those that exploit the spin of electrons rather than their charge-rely on effects that produce, control, and detect spin-polarized currents. Many of these schemes exploit the Edelstein effect, in which an electrical current passing through a material induces a spin polarization. Recent studies have highlighted a weakness of most models of the Edelstein effect: They neglect contributions from an electron's orbital motion, assuming that they are much smaller than those from an electron's spin. But calculations showed that, in bulk materials and at metal surfaces, these contributions can sometimes dwarf the spin contributions. Now, a theory developed by Annika Johansson at the Institute of Physics in Halle, Germany, and colleagues shows that orbital contributions also dominate the Edelstein effect in a two-dimensional metal oxide [1].

Build a layered structure made of a nanometer-thick oxide film deposited on a strontium titanate (STO) substrate, and a

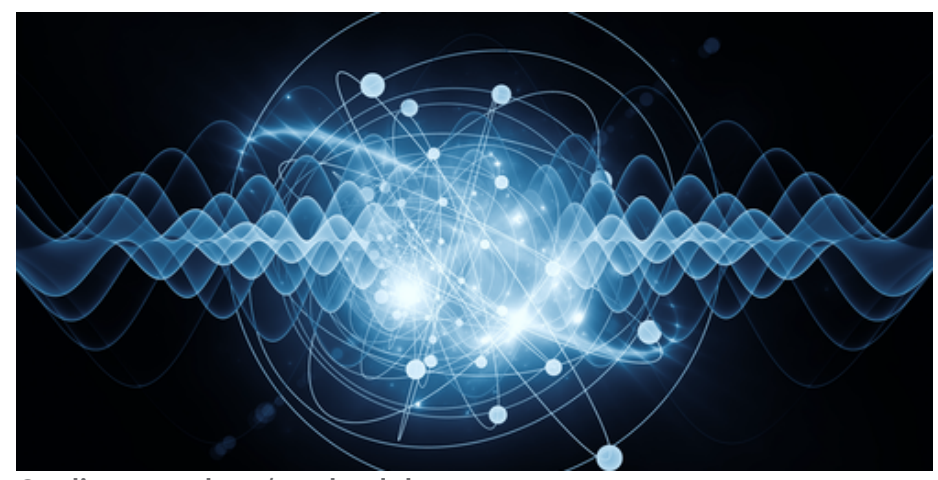

Credit: agsandrew/stock.adobe.com two-dimensional gas of electrons (2DEG) will form at the interface between the two materials. Previous studies indicate that this 2DEG has a host of promising features for spintronics, including high mobility, tunable carrier density, giant spin-orbit coupling, and immunity from scattering. But to use STO in devices, researchers need a better understanding of the Edelstein effect in these structures, which is where the results from Johansson and her colleagues come in.

The team examined the detailed band structure of the 2DEG at an STO interface. They found that the orbital contribution to the Edelstein effect is more than 10 times greater than the spin contribution. They say that this large orbital Edelstein effect could be harnessed to demonstrate a recently proposed magnetoelectric spintronic device that could offer an energy-efficient alternative to the conventional transistors used in today's electronics.

Correction (1 April 2021): An earlier version failed to mention previous calculations suggesting a dominating orbital contribution to the Edelstein effect in bulk materials and at metal surfaces.

Matteo Rini is the Editor of Physics.

\section{REFERENCES}

1. A. Johansson et al., "Spin and orbital Edelstein effects in a two-dimensional electron gas: Theory and application to $\mathrm{SrTiO}_{3}$ interfaces," Phys. Rev. Research 3, 013275 (2021). 